\title{
Cardio-Respiratory Events and Food Autonomy Responses to Early Uni-Modal Orofacial Stimulation in Very Premature Babies : A Randomized, Controlled Study
}

\section{Sahra Meziane}

Department of Neonatology, Hôpital Nord, Assistance Publique-Hôpitaux de Marseille, Véronique Brévaut-Malaty

Department of Neonatology, Hôpital Nord, Assistance Publique-Hôpitaux de Marseille, Aurélie Garbi

Department of Neonatology, Hôpital Nord, Assistance Publique-Hôpitaux de Marseille, Muriel Busuttil

Department of Neonatology, Hôpital Nord, Assistance Publique-Hôpitaux de Marseille,

\section{Gaelle Sorin}

Department of Neonatology, Hôpital Nord, Assistance Publique-Hôpitaux de Marseille,

Barthélémy Tosello ( $\square$ barthelemy.tosello@ap-hm.fr)

Department of Neonatology, Hôpital Nord, Assistance Publique-Hôpitaux de Marseille,

\section{Catherine Gire}

Department of Neonatology, Hôpital Nord, Assistance Publique-Hôpitaux de Marseille,

\section{Research Article}

Keywords: Cardio-respiratory, Uni-Modal Orofacial Stimulation, Randomized, cardiorespiratory

Posted Date: December 28th, 2020

DOl: https://doi.org/10.21203/rs.3.rs-129410/v1

License: (c) (i) This work is licensed under a Creative Commons Attribution 4.0 International License. Read Full License

Version of Record: A version of this preprint was published at Children on December 16th, 2021. See the published version at https://doi.org/10.3390/children8121188. 


\section{Abstract}

\section{Background}

The Uni-modal OroFacial Stimulation (OFS) for preventing very preterm infants' oral disorders is highly controversial. Our study sought to demonstrate that OFS reduced cardio-respiratory events and improved food autonomy in a population of very preterm infants.

\section{Methods}

Randomized, controlled, prospective, and unicentric study. Preterm included were born between 26-29 week's gestational age (GA) and with a corrected postnatal age $<33$ week's GA. They were randomized in two groups: experimental group underwent an OFS according to a protocol established over 10 consecutive days, and the control group underwent no OFS. The primary outcome were the number of cardiorespiratory events: apnea-bradycardia (with or without desaturations) or number of isolated desaturations which were evaluated at four separate times. The measurement occurred during a first, four and eight independent feedings.

Results

17 patients in the experimental group and 18 in the control group were included. The number of cardiorespiratory events for all independent- feedings time was significantly reduced in the OFS group ( $p$ $=0.003)$ in the univariate analysis but not in multivariable analysis. There were no signs of poor tolerance noted in the protocol. The quantity of milk ingested during the first autonomous feeding was higher in the experimental group. The acquisition of food autonomy and the duration of the hospitalization stay were similar in the two groups.

\section{Conclusions}

While our study does not affirm that an early unimodal OFS improves the premature infants' cardiorespiratory evolution and/or the acquisition of food autonomy, it does indicate an improved food efficiency during their first autonomous feedings.

The ClinicalTrials.gov identifier is NCT01116765, on May 2010.

\section{Background}

The transition from enteral to autonomous oral feeding is a fundamental concern for both parents and caregivers, since it is a determinant for the length of the preterm neonate's hospital stay. Premature infants ( $<37$ weeks GA) are at risk for feeding difficulties and have a delay in achieving oral food intake autonomy. There can be an early oral disorder in the context of artificial nutrition [1]. Oral feeding requires coordination of sucking, swallowing and breathing. This coordination is not mature until 32 weeks GA [2]. 
In the hospital setting, it is the coordination of sucking in conjunction with swallowing and respiratory control that will ultimately lead to "preparation for oral feeding" in a safe and effective manner [3].

A distinction is made between nutritive sucking (NS) when ingesting milk, and non-nutritive sucking (NNS) without ingestion of liquid (nipple or dummy). NNS offers certain benefits that are not directly involved in oral feeding [4-7]. These include promoting weight gain, producing an analgesic effect, reducing stress, stabilizing the child's behavior and accelerating the progression of oral nutrition. Additionally, it is a good marker of the level of maturation of sucking itself. Factors which help promote the maturation of NNS include as well as oral stimulation through NNS or sensorimotor stimulation of the oral structures, performed during enteral feeding of clinically stable premature infants over 30 Weeks GA [8].

In 2002, Fucile et al. reported that there was a more rapid acquisition of food autonomy after 10 days of unimodal oral stimulation, with no differences found over the duration of hospitalization [8]. Recently, a review of the Cochrane library [9] suggested a decrease in the transition time to autonomous oral feeding and a shorter hospital stay with a NNS.

On the other hand, no study found any effect on the presence of cardio-respiratory events while improving food autonomy or weight gain. However, the studies are methodologically heterogeneous, thus making it difficult to interpret the results. This calls into question the usefulness of these interventions in current practice.

Our study's objectives were to demonstrate that early NNS unimodal OroFacial Stimulation (OFS) reduces apnea-bradycardia and/or desaturations, and improves food autonomy in very premature infants born between 26 and 29 Weeks GA.

\section{Methods}

\section{Design}

This study was monocentric, controlled, open, and randomized into two groups. The recruitment was prospective. The experimental group was represented by newborns receiving an OFS associated with enteral nutrition based on a program inspired by Bruwier and Fucile $[8,12]$. The control group corresponded to newborns having an enteral feeding without receiving any OFS. Both groups received NNS from a pacifier.

The ClinicalTrials.gov identifier was NCT01116765, on 05 May 2010 (https://www.clinicaltrials.gov/ct2/show/NCT01116765?term=NCT01116765\&draw=2\&rank=1).

\section{Sample}

The inclusion criteria were : 1 neonate born between 26 and 29 completed Week's GA, 2 less or equal 33 Week's GA corrected age (CA), 3 hospitalized in our neonatal unit, 4 without neurological pathologies; 
confirmed by a normal fontanellar ultrasound or grade 1 or 2 intraventricula hemorrhage [10] as well as a normal cerebral MRI or type 1 to 4 brain abnormalities according to the modified Paneth classification [11], 5 well-tolerated enteral diet, greater than $100 \mathrm{ml} / \mathrm{kg} / \mathrm{day}, 6$ free from infectious pathologies making them clinically unstable (reactive $C$ protein less than $7 \mathrm{mg} / \mathrm{L}$ ), 7 any CPAP (Control Positive Airway Pressure) for at least 48 hours, 8 had no congenital anomalies and 8 finally, both parents and their legal representatives agreed to participate in the study and signed an informed consent.

The exclusion criteria were: 1) children for whom one of the parents/legal guardians had withdrawn consent before the end of the study, 2) children with co-morbidity during the stimulation period requiring the sessions to be stopped (nosocomial infection and/or ulcerative-necrotizing enteritis and/or respiratory worsening requiring cessation of feeding and/or resumption of CPAP and/or an intensive care admission, 3) children transferred to another establishment before the end of the program, 4) children whose program sequence was not fully completed, and 5) deceased children.

The total duration of inclusion was set at 18 months.

\section{Intervention}

The program included 10 consecutive days of OFS for the very preterm newborn. Twice a day, for 10 consecutive days, a 15 to 20 minutes sensorimotor tactile stimulation was performed 15 to 30 minutes before feeding by gastric tube. The caretaker performed a careful handwashing and gloving prior to each session. The first 12 minutes of stimulation involved the cheeks, lips, gums and tongue, and the last 3 minutes consisted of suction stimulation (NNS).

The children were calm and awake in either their crib or incubator and placed in a semi-seated position. The pediatric nurse or parent supported the child's head and assumed an enveloping and secure position. No stimulation was performed if the child was in deep sleep, showed signs of discomfort or fatigue. This was evaluated by nurses who had been trained in NIDCAP development care and used the Newborn Individualized Developmental Care and Assessment Program [NIDCAP] grid [13, 14].

\section{Setting}

This centrally administered trial was conducted in a level 3 maternity unit from Marseille in France and included neonates born between January 2013 and December 2014. In the study hospital, there are 2,700 live births per year, $8 \%$ of which are very preterm births.

\section{Measurements}

The primary endpoint was composite and included the evaluation of apnea-bradycardia (with or without associated desaturations). It additionally included the evaluation of isolated desaturations during the learning period towards food autonomy with an analysis at four different times: 1) at the first autonomous feeding, 2) up to four independent feedings, 3) up to eight independent feedings on 2 consecutive days, and 4) and then all these autonomous feedings combined. 
Apnea-bradycardia with or without desaturation corresponded to a respiratory pause $>10$ seconds associated with bradycardia $<80$ beats per minute, associated, or not, with desaturation $<80-85 \%$ on the pulse oximeter. An isolated desaturation corresponded to an oxygen saturation $<90 \%$. The monitoring of the cardio-respiratory manifestations of the children of the two groups was carried out from their inclusion in the study to their discharge from the neonatology department. The vital constants were assessed in real time or by retrospectively analysing traces recorded by the scope. Cardio-respiratory manifestations were recorded 30 minutes before, during, and three hours after each feeding.

The secondary outcomes included

- A validation of our OFS protocol by evaluating its tolerance and listing the cardio-respiratory manifestations (apneas, bradycardias and desaturations at all feeds from D1 to D10 of the stimulation program, always 30 minutes before, during and in the hour after feeding).

- The acquisition of food autonomy, which was defined when the newborn achieved eight independent feedings for two consecutive days.

- The date of the first autonomous feeding.

- The amount of milk ingested.

- Weight increase.

- The length of hospital stay.

- Dietary monitoring at six months corrected age.

- The quality of life of the child, assessed by the parents, using the QUALIN questionnaire [15], at six and 12 months of corrected age.

\section{Statistical analyses}

Randomization was established using the OLAN procedure (SAS software), in order to divide the participants into two parallel and comparable groups. Data analysis was performed using SPSS software. The qualitative variables were presented in the form of percentages and the quantitative variables were in the form of mean \pm standard deviation. The normality of the distributions of the quantitative data was systematically sought using the Shapiro-Wilks test. If several variables were not normally distributed, usual transformation techniques (logarithmic, etc.) or normalization algorithms (Blom or Tukey algorithms) were used to measure the interpretation of the results that resulted.

The statistical tests used were: The Student test or the non-parametric Mann-Whitney test for comparing means, the median test, Chi2 test or Fisher's exact test for qualitative variables All tests were bilateral. The median and the mean were close for all quantitative variables. 
The number of subjects required was calculated for the main composite criterion: the number of apneabradycardias (with or without associated desaturations) and the number of desaturations. To highlight a decrease of at least two apnea-bradycardias or two desaturations during the four different times of autonomous feedings, it was evaluated with 21 patients by group with a risk of error of $a=0.05$ and an expected power of $80 \%$. The significance threshold was fixed at $p=0.05$ in a bilateral situation.

The primary and secondary endpoint analyses were done on an intention-to-treat basis. The multivariate analyses consisted of modelling the primary and secondary endpoints, according to the two study groups, with parameters: gestational age, birth weight and total ventilation time (invasive and noninvasive).

\section{Results}

\section{Characteristic of sample}

There were 53 patients included and randomized. Of the 35 who were not exluded, 17 were from the experimental group with OFS and 18 were from the control group without OFS.

During the 12-month follow-up, eight patients were lost to follow-up: four from the experimental group and four from the control group (Figure 1).

There were 11 boys (64.7\%) in the "with OFS" group and nine boys (50\%) in the "without OFS" group. The mean term of birth was 28 weeks GA in the two groups. The average birth weight was $1101 \mathrm{~g}$ in the experimental group and $1047 \mathrm{~g}$ in the control group.

The baseline age at inclusion was 16.5 days ( 30 weeks $\mathrm{GA}$ and 2 days corrected age) in the experimental group, and 18 days, (30 weeks GA and 4 days corrected age) in the control group. There were no significant differences between the two groups and both groups were comparable in terms of all their antenatal, neonatal and postnatal characteristics (Table 1).

\section{Primary objective}

The cardio-respiratory manifestations were significantly lower in the experimental group compared with control group (respectively $n=102$ vs $n=61, p=0.003$ ) but the differences observed between the two groups was no longer significant $(p=0.39)$ after adjustment with gestational age, birth weight and total duration of ventilation.

We noted no statistically significant differences between the two groups when detailing apneabradycardia with or without associated desaturations, and isolated desaturations during the four different times of the analysis (1, 4, and 8 autonomous feedings or all these feedings combined [Table 2]).

\section{Secondary objectives}


There appeared to be no intolerance to the OFS protocol. The weight gain in both groups appeared similar.

Although not significant $(p=0.70)$, the experimental group's infants had a higher weight when discharged $(2877( \pm 366) \mathrm{g})$ than the control group $(2821( \pm 474) \mathrm{g})$. The total hospital stay length was shorter in the OFS group than the control group (respectively: $76.18( \pm 15.88)$ days vs $78.72( \pm 17.67)$ days, $p=0,66)$ (Table 3).

The completion date for the eight autonomous feedings, continuing for 48 consecutive hours, which represented the date of acquisition of food autonomy, was earlier in the experimental group (corrected age of $36.41( \pm 1.06)$ Weeks GA) than in the control group (36.78 ( \pm 1.44$)$ Weeks GA), but the difference was not significant $(\mathrm{p}=0.40)$ (Figure 2).

Only the quantity of milk ingested during the first autonomous feeding was significantly higher in the experimental group than in the control group (respectively $34.11 \mathrm{ml}(+/-6.60)$ vs $29.50 \mathrm{ml}(+/-5,94), \mathrm{p}=$ $0.04)$, in the univariate analysis. In the multivariate analysis with the adjustment on the parameters (gestational age, birth weight and total ventilation time), the observed difference between the two groups was no longer significant, however close to being significant $(p=0.07)$ (Figure 3 ).

A six-month nutritional follow-up concerning the during of breast feedings, achievement of diversification and the presence of a normal gag reflex, was comparable in the two groups. The infant's quality of life at 6 and 12 months was evaluated using the QUALIN questionnaire by the parents and showed no significant difference between the two groups (Table 4).

\section{Discussion}

Our study's results suggest that early unimodal oral sensorimotor tactile stimulation does not significantly improve the cardio-respiratory stability or food autonomy of very premature babies.

No effect was found on the quantity of milk ingested (except at the first feeding), weight gain, or the length of their hospital stay. The nutritional outcome at six months was unchanged, as was the quality of life of the child at six and 12 months as assessed by the parents.

Similar to our study, a recent meta-analysis studied the effects of NNS in preterm infants under 37 Weeks GA and found no significant effect on the decrease in cardio-respiratory events [16].

On the other hand, our results concerning food autonomy are not consistent with most of the literature. Numerous studies suggested that the duration between the transition from enteral feeding to autonomous oral feeding was reduced through oral stimulation [17-22]. Our study showed a greater quantity of milk ingested during the first feeding which remained within the limits of significance. The average reduction time found in the different studies was 5 to 10 days. Rocha et al, in their randomized trial of 98 premature infants between 26 and 32 weeks GA found a more rapid 9-day acquisition of food autonomy [23]. Tian et al, in their meta-analysis of 855 newborns between 27 and 33 weeks of age, found 
a similar result, with a decrease of around four days [24]. Lessen et al found that for 20 premature newborns from 29 weeks GA, the decrease was five days [25] As in our study, the correlation was even more marked when the protocols used combined sensorimotor oral stimulation with NNS [26, 27].

Finally, the results concerning the effect of oral stimulation on the length of hospital stays are heterogeneous [21, 28]. For Rocha et al, as well as Tian et al, a significant reduction in the length of the hospital stay is observed to be between four to ten days. For Lessen et al, the effect of oral stimulation on the length of hospital stay was not found.

Other studies did not find any significant differences either on food autonomy or on the length of the hospital stay [28]. This is also the case with the study by Bache et al, which showed an increase in the rate of breastfeeding on discharge from the hospital, which was not the case in our study [29].

Our study's strengths included its randomized nature, the presence of a comparable control group, the analysis carried out with an intention-to-treat basis, and the analysis of multiple parameters (secondary criteria). Strengths also included the frequently used OFS oral protocols, which resulted from Bruwier's and Fucile's work, and for which no signs of intolerance were noted. The study's paramedical staff received OFS training by qualified professional staff and the inclusion of a neonatology speech therapist was an additional asset. We were able to avoid confounding factors that can modify orality by using multivariate analyses with adjustment for gestational age, birth weight and total ventilation time. Finally, we were able to take into account the quality of life of children as assessed by the parents in the medium term.

Our study's limitations were represented by the absence of a blind methodology as well as the unicentric nature of the trial. The large number of people lost to follow-up minimized the number of necessary subjects, and certain parameters at the limit of significance could have been improved. When the infant received oral stimulation by different personnel such as childcare workers or childcare assistants, the reproducibility of the sessions may not have been similar. For hygienic reasons, the personnel's gloved finger used might have been a source of discomfort for the child. We were unable to measure the quantitative parameters of the milk suction rate transfer, or the quantity of milk ingested during the first five minutes, on the total milk consumption or even the nutritional efficiency. Studies having analyzed the sucking measurement parameters [30-32], note an improvement with oral stimulation, in particular the rate of milk transfer (quantity of milk ingested/duration of feeding in millilitres per minute) as well as the sucking frequency. This appears especially true since the quantity of milk ingested during first autonomous feed is at the limit of significance in multivariate analysis.

A major study weakness was the lack of details concerning OFS sessions which had no parent present. It can easily be assumed that when parents were present during the sessions, the newborn had conditions which enhanced their tolerance and well-being.

Finally, with improved developmental care for newborns, initiation to oral feeding should be done on the basis of personal observation for each newborn and not systematically from their given gestational age. 
For individual adaptation, quantitative evaluation scores of nutritive suction make it possible to determine if the child is ready for this initiation to oral feeding [33].

Research shows that the prematurely born child's development takes place more in a dynamic of multisensory rather than single-sensory stimulation. Weekly parent-administered massage therapy, when combined with visual interaction between the parents and children, results in a more rapid acquisition of food autonomy. There is often a two-fold increase in breastfeeding rates at discharge, including reduced maternal stress, and a likely increased breastfeeding efficiency [34-36], suggest that vagal stimulation resulting from combined tactile and kinesthetic stimulation allows an increase in gastric motility which leads to an increase in weight gain. Also, multisensory intervention: auditory, visual, tactile and vestibular, stimulates brain development by modifying the cerebral structure of the child. This allows the maturation of food capacities by increasing the number of sucks and the average number of sucks per burst, after performing this intervention after 32 weeks of GA corrected age, in newborns between 29 and 34 weeks of age $[38,39]$.

Finally three studies compared multimodal and unimodal stimulation and suggested: 1 ) that a combined oral and tactile stimulation program would be more effective than a tactile stimulation program alone, for the duration of acquisition of food autonomy, weight gain and length of hospital stay for premature infants [40], 2) multimodal oral, tactile and kinaesthetic stimulation would improve food efficiency compared to unimodal stimulation: oral or tactile/kinaesthetic [41), and 3) multimodal stimulation by NNS and auditory stimulation would shorten the transition period between autonomous enteral and oral feeding as compared to NNS alone [42]. This phenomenon could be explained by the synergistic effect of multimodal stimulation on food parameters.

\section{Conclusion}

Early non-nutritive OFS does not reduce cardio-respiratory events during the period of acquisition of food autonomy, nor does it reduce the duration of acquisition of food autonomy, but it may improve nutritional efficiency. The results on this subject remain controversial in the literature. Continued studies on the subject with a more robust methodology, and with a dynamic analysis of sucking measurements, multimodal stimulation seem appropriate.

\section{Abbreviations}

Uni-modal OroFacial Stimulation (OFS); gestational age (GA); nutritive sucking (NS); non-nutritive sucking (NNS); corrected age (CA); CPAP (Control Positive Airway Pressure).

\section{Declarations}

\section{Ethics approval and consent to participate}


The protocol was approved by the institutional ethics committee and by the French Protection Committee ( $N^{\circ}$ IDRCB 2009-A01191-56, 1 January 2010), by the French national institution of pharmacovigilance (ANSM, Agence Nationale de Sécurité du Médicament et des produits de santé) (27 November 2009). Informed consents were obtained once the information leaflet was reviewed with the parents or legal representatives of the children. Participants were advised they could withdraw their consent at any time, and that the data would be kept confidential.

\section{Availability of data and materials}

The data supporting the current findings are not publicly available since the database is currently not anonymous and contains all the patients' names. However, it will be available upon request.

\section{Competing interests}

The authors declare that they have no competing interests.

\section{Funding}

This work was supported by institutional grants from the French 2009 Appel d'Offre Recherche Clinique Assistance Publique, Hôpitaux de Marseille. The sponsor was represented by Assistance Publique, Hôpitaux de Marseille, France, whose role was to control the appropriateness of ethical and legal considerations.

\section{Authors' contributions}

SM, CG, VBM and BT contributed to conception and design of the manuscript, clinical management of the patient, and drafted the manuscript. SM, VBM and CG contributed to acquisition, analysis and interpretation of data, and drafting of the manuscript. AG, MB, GS and BT contributed to critical revision of the manuscript for important intellectual content. All authors read and approved the final version of the manuscript.

\section{Acknowledgements}

The authors would like to thank the parents for allowing their infants to participate in the study, as well as the nursing staff and the nurse supervisor for her valued help.

\section{References}

[1]. Marret S, Chollat C, de Quelen R, Pinto Cardoso G, Abily-Donval L, Chadie A, et al. Course and neurological/behavioral development of preterm children. 2015;22(2):195-202.

[2]. Lau C, Smith E, Schanler R. Coordination of suck-swallow and swallow respiration in preterm infants. Acta Paediatr. 2007;92(6):721-7. 
[3]. Lau C, Smith EO. A Novel Approach to Assess Oral Feeding Skills of Preterm Infants. Neonatology. 2011;100(1):64-70.

[4]. Pinelli J, Symington AJ. Non-nutritive sucking for promoting physiologic stability and nutrition in preterm infants. Cochrane Database Syst Rev. 2005;19;(4):CD001071.

[5]. Pineda R, Dewey K, Jacobsen A, Smith J. Non-Nutritive Sucking in the Preterm Infant. Am J Perinatol. 2019;36(03):268-76.

[6]. Harding C, Frank L, Van Someren V, Hilari K, Botting N. How does non-nutritive sucking support infant feeding? Infant Behav Dev. 2014;37(4):457-64.

[7]. Bernbaum JC, Pereira GR, Watkins JB, Peckham GJ. Nonnutritive Sucking During Gavage Feeding Enhances Growth and Maturation in Premature Infants. Pediatrics. 1983;71(1):41-5.

[8]. Fucile S, Gisel E, Lau C. Oral stimulation accelerates the transition from tube to oral feeding in preterm infants. J Pediatr. 2002;141(2):230-6.

[9]. Greene Z, O'Donnell CP, Walshe M. Oral stimulation for promoting oral feeding in preterm infants. Cochrane Database Syst Rev. 2016;9(9):CD009720.

[10]. Papile L-A, Burstein J, Burstein R, Koffler H. Incidence and evolution of subependymal and intraventricular hemorrhage: A study of infants with birth weights less than 1,500 gm. J Pediatr. 1978;92(4):529-34.

[11]. Paneth N. Classifying brain damage in preterm infants. J Pediatr. 1999;134(5):527-9.

[12]. Fucile S, Gisel E, Lau C. Effect of an oral stimulation program on sucking skill maturation of preterm infants. Dev Med Child Neurol. 2005;47(3):158-62.

[13]. Als H, Tronick E, Lester BM, Brazelton TB. The Brazelton Neonatal Behavioral Assessment Scale (BNBAS). J Abnorm Child Psychol. 1977;5(3):215-29.

[14]. Wallin L, Eriksson M. Newborn Individual Development Care and Assessment Program (NIDCAP): A Systematic Review of the Literature. Worldviews Evid Based Nurs. 2009;6(2):54-69.

[15]. Manificat S, Dazord A, Langue J, Danjou G, Bauche P, Bovet F, et al. Evaluation de la qualité de vie du nourrisson et du très jeune enfant: Validation d'un questionnaire. Étude multicentrique européenne. Arch Pediatr. 2000;7(6):605-14.

[16]. Foster JP, Psaila K, Patterson T. Non-nutritive sucking for increasing physiologic stability and nutrition in preterm infants. Cochrane Database Syst Rev. 2016 Oct 4;10(10):CD001071.

[17]. Ghomi H, Yadegari F, Soleimani F, Knoll BL, Noroozi M, Mazouri A. The effects of premature infant oral motor intervention (PIOMI) on oral feeding of preterm infants: A randomized clinical trial. Int $\mathrm{J}$ 
Pediatr Otorhinolaryngol. 2019;120:202-9.

[18]. Fucile S, Milutinov M, Timmons K, Dow K. Oral Sensorimotor Intervention Enhances Breastfeeding Establishment in Preterm Infants. Breastfeed Med. 2018;13(7):473-8.

[19]. Arora K, Goel S, Manerkar S, Konde N, Panchal H, Hegde D, et al. Prefeeding Oromotor Stimulation Program for Improving Oromotor Function in Preterm Infants - A Randomized Controlled Trial. Indian Pediatr. 2018;55(8):675-8.

[20]. Bala P, Kaur R, Mukhopadhyay K, Kaur S. Oromotor stimulation for transition from gavage to full oral feeding in preterm neonates: A Randomized controlled trial. Indian Pediatr. 2016;53(1):36-8.

[21]. Lima AH, Côrtes MG, Bouzada MCF, Friche AA de L. Preterm newborn readiness for oral feeding: systematic review and meta-analysis. CoDAS. 2015;27(1):101-7.

[22]. Simpson C, Schanler RJ, Lau C. Early Introduction of Oral Feeding in Preterm Infants. Pediatrics. 2002;110(3):517-22.

[23]. Rocha AD, Moreira MEL, Pimenta HP, Ramos JRM, Lucena SL. A randomized study of the efficacy of sensory-motor-oral stimulation and non-nutritive sucking in very low birthweight infant. Early Hum Dev. 2007;83(6):385-8.

[24]. Tian X, Yi L-J, Zhang L, Zhou J-G, Ma L, Ou Y-X, et al. Oral Motor Intervention Improved the Oral Feeding in Preterm Infants: Evidence Based on a Meta-Analysis With Trial Sequential Analysis. Medicine (Baltimore). 2015;94(31):e1310.

[25]. Lessen BS. Effect of the Premature Infant Oral Motor Intervention on Feeding Progression and Length of Stay in Preterm Infants: Adv Neonatal Care. 2011;11(2):129-39.

[26]. Zhang Y, Lyu T, Hu X, Shi P, Cao Y, Latour JM. Effect of Nonnutritive Sucking and Oral Stimulation on Feeding Performance in Preterm Infants: A Randomized Controlled Trial. Pediatr Crit Care Med. 2014;15(7):608-14.

[27]. Arvedson J, Clark H, Lazarus C, Schooling T, Frymark T. Evidence-Based Systematic Review: Effects of Oral Motor Interventions on Feeding and Swallowing in Preterm Infants. Am J Speech Lang Pathol. 2010;19(4):321-40.

[28].Thakkar PA, Rohit HR, Ranjan Das R, Thakkar UP, Singh A. Effect of oral stimulation on feeding performance and weight gain in preterm neonates: a randomised controlled trial. Paediatr Int Child Health. 2018;1-6.

[29]. Bache M, Pizon E, Jacobs J, Vaillant M, Lecomte A. Effects of pre-feeding oral stimulation on oral feeding in preterm infants: A randomized clinical trial. Early Hum Dev. 2014;90(3):125-9. 
[30]. Li X-L, Liu Y, Liu M, Yang C-Y, Yang Q-Z. Early Premature Infant Oral Motor Intervention Improved Oral Feeding and Prognosis by Promoting Neurodevelopment. Am J Perinatol. 2019;s-0039-1685448.

[31]. Grassi A, Sgherri G, Chorna O, Marchi V, Gagliardi L, Cecchi F, et al. Early Intervention to Improve Sucking in Preterm Newborns: A Systematic Review of Quantitative Studies. Adv Neonatal Care. 2019;19(2):97-109.

[32]. Aguilar-Rodríguez M, León-Castro JC, Álvarez-Cerezo M, Aledón-Andújar N, Escrig-Fernández R, Rodríguez de Dios-Benlloch JL, et al. The Effectiveness of an Oral Sensorimotor Stimulation Protocol for the Early Achievement of Exclusive Oral Feeding in Premature Infants. A Randomized, Controlled Trial. Phys Occup Ther Pediatr. 2019;1-13.

[33]. Neiva F, Leone C, Leone C. Non-nutritive sucking scoring system for preterm newborns. Acta Paediatr. 2008;97(10):1370-5.

[34]. Fontana C, Menis C, Pesenti N, Passera S, Liotto N, Mosca F, et al. Effects of early intervention on feeding behavior in preterm infants: A randomized controlled trial. Early Hum Dev. 2018;121:15-20.

[35]. Newnham CA, Milgrom J, Skouteris H. Effectiveness of a Modified Mother-Infant Transaction Program on Outcomes for Preterm Infants from 3 to 24 months of age. Infant Behav Dev. 2009;32(1):17-26.

[36]. Vickers A, Ohlsson A, Lacy J, Horsley A. Massage for promoting growth and development of preterm and/or low birth-weight infants. Cochrane Database Syst Rev. 2004;2004(2):CD000390.

[37]. Field T, Ignatoff E, Stringer S, Brennan J, Greenberg R, Widmayer S, et al. Nonnutritive Sucking During Tube Feedings: Effects on Preterm Neonates in an Intensive Care Unit. Pediatrics. 1982;70(3):381-4.

[38]. Medoff-Cooper B, Rankin K, Li Z, Liu L, White-Traut R. Multisensory Intervention for Preterm Infants Improves Sucking Organization: Adv Neonatal Care. 2015;15(2):142-9.

[39]. Kanagasabai PS, Mohan D, Lewis LE, Kamath A, Rao BK. Effect of Multisensory Stimulation on Neuromotor Development in Preterm Infants. Indian J Pediatr. 2013;80(6):460-4.

[40]. Gaebler CP, Hanzlik JR. The Effects of a Prefeeding Stimulation Program on Preterm Infants. Am J Occup Ther. 1996;50(3):184-92.

[41]. Fucile S, Gisel EG, Mcfarland DH, Lau C. Oral and non-oral sensorimotor interventions enhance oral feeding performance in preterm infants: Oral Feeding Following Sensorimotor Interventions. Dev Med Child Neurol. 2011;53(9):829-35.

[42]. Standley JM, Cassidy J, Grant R, Cevasco A, Szuch C, Nguyen J, et al. The effect of music reinforcement for non-nutritive sucking on nipple feeding of premature infants. Pediatr Nurs. 2010;36(3):138-45. 


\section{Tables}

Tableau 1. Characteristics of study population.

\begin{tabular}{|c|c|c|c|}
\hline Characteristics & $\begin{array}{l}\text { With OFS } \\
(n=17)\end{array}$ & $\begin{array}{l}\text { Without OFS } \\
(n=18)\end{array}$ & $\mathrm{p}$ \\
\hline \multicolumn{4}{|l|}{ ANTENATAL } \\
\hline Maternal age (years), Mean ( \pm SD) & $31.94( \pm 5.2)$ & $28.89( \pm 7.1)$ & 0.15 \\
\hline Twin Pregnancies, n (\%) & $4(23.5)$ & $4(22.2)$ & 1.00 \\
\hline Pregnancies with pathology, n (\%) & $6(35.3)$ & $4(22.2)$ & 1.00 \\
\hline Intrauterine Growth Retardation, n (\%) & $0(0)$ & $2(11.1)$ & 0.48 \\
\hline Cesarean Births, n (\%) & $12(70.6)$ & $14(77.8)$ & 0.47 \\
\hline \multicolumn{4}{|l|}{ NEONATAL } \\
\hline Weeks GA $(\mathrm{GA})$, average $( \pm \mathrm{SD})$ & $27.98( \pm 1.3)$ & $28.07( \pm 1.3)$ & 0.55 \\
\hline Males, n (\%) & $11(64.7)$ & $9(50)$ & 0.50 \\
\hline Average birth weight $(\mathrm{g})$, Mean $( \pm S D)$ & $\begin{array}{l}1001.76 \\
( \pm 224.7)\end{array}$ & $\begin{array}{l}1046.94 \\
( \pm 181.8)\end{array}$ & 0.56 \\
\hline SGA, n (\%) & $1(5.9)$ & $3(16.7)$ & 0.34 \\
\hline \multicolumn{4}{|l|}{ POSTNATAL } \\
\hline $\begin{array}{l}\text { Week Corrected Gestationnel Age at inclusion }(G A) \text {, } \\
\text { mean }( \pm \text { SD) }\end{array}$ & $30.37( \pm 1.1)$ & $30.60( \pm 1.0)$ & 0.41 \\
\hline Age at inclusion (days), mean ( \pm SD) & $16.6( \pm 9.8)$ & $17.8( \pm 11.5)$ & 0,92 \\
\hline Parenteral feeding time (days), mean ( $₫ S D$ ) & $21.1( \pm 9.6)$ & $23.7( \pm 9.6)$ & 0.42 \\
\hline Total ventilation time (days), mean ( \pm SD) & $22.9( \pm 13,4)$ & $21( \pm 12)$ & 0.73 \\
\hline Total invasive ventilation time (days mean $( \pm S D$ ) & $5.7( \pm 6.9)$ & $4( \pm 3.1)$ & 0.81 \\
\hline Non-invasive ventilation time (days), mean ( \pm SD) & $18.2( \pm 8.4)$ & $17.8( \pm 10.6)$ & 0.74 \\
\hline Weight at inclusions (grams), mean ( \pm SD) & $1471( \pm 196)$ & $1449( \pm 274)$ & 0.79 \\
\hline
\end{tabular}

OFS: oro-facial stimulation; GA: gestational age; SGA : Small for Gestationnel Age.

Data are presented as $\mathrm{n}(\%)$ unless stated differently. $\mathrm{P}$ value of the difference between groups with available data, $p<0.05$ : statistically significant difference. 
Table 2. Number of apnea-bradycardia (with or without desaturations), isolated desaturations and all cardiorespiratory, events combined, in experimental versus control group, during transition to food autonomy.

\begin{tabular}{|c|c|c|c|c|}
\hline $\begin{array}{l}\text { With OFS } \\
(n=17)\end{array}$ & $\begin{array}{l}\text { Without OFS } \\
(n=18)\end{array}$ & $\mathrm{p}^{\mathrm{a}}$ & ß & $p^{b}$ \\
\hline \multicolumn{5}{|c|}{ Number of apnea-bradycardia ( \pm desaturations) per patient, mean $( \pm S D$ ) } \\
\hline $0.59( \pm 0.80)$ & $0.33( \pm 0.77)$ & 0.34 & & \\
\hline $0.82( \pm 1.29)$ & $1.18( \pm 1.55)$ & 0.48 & & \\
\hline $1.94( \pm 2.77)$ & $3.89( \pm 4.97)$ & 0.16 & & \\
\hline $3.24( \pm 3.55)$ & $5.33( \pm 6.04)$ & 0.22 & 1.21 & 0.50 \\
\hline \multicolumn{5}{|c|}{ Number of isolated desaturations per patient, mean $( \pm S D)$} \\
\hline $0( \pm 0)$ & $0.17( \pm 0.38)$ & 0.08 & & \\
\hline $0.59( \pm 1.37)$ & $0.41( \pm 1.70)$ & 0.74 & & \\
\hline $0.81( \pm 1.76)$ & $1.17( \pm 2.20)$ & 0.61 & & \\
\hline $1.35( \pm 2.71)$ & $1.72( \pm 2.54)$ & 0.68 & 0.55 & 0.56 \\
\hline \multicolumn{5}{|c|}{$\begin{array}{l}\text { Number of feeds with cardiorespiratory events (all combined)/Number of feeds without } \\
\text { cardiorespiratory events }\end{array}$} \\
\hline $61 / 271$ & $102 / 265$ & $0.003^{*}$ & 1.53 & 0.39 \\
\hline
\end{tabular}

OFS: oro-facial stimulation; SD: standard deviation.

$p$ value of the difference between the groups with available data

a Value of $p$ in univariate analysis.

b Value of $p$ in multivariate analysis including the parameters: gestational age, birth weight and total duration of ventilation.

${ }^{*} p<0.05$ : statistically significant difference.

ß: correlation coefficient in multivariate analysis.

Table 3. Evaluation: tolerance of OFS protocol (Tolerance Day 1 to Day 10). 


\begin{tabular}{|c|c|c|c|c|c|}
\hline & $\begin{array}{l}\text { With OFS } \\
(n=17)\end{array}$ & $\begin{array}{l}\text { Without OFS } \\
(n=18)\end{array}$ & $\mathrm{p}^{\mathrm{a}}$ & ß & $\mathrm{p}^{\mathrm{b}}$ \\
\hline Number of apneas & $3.13( \pm 6.15)$ & $3.17( \pm 5.85)$ & 0.99 & -0.69 & 0.65 \\
\hline Number of bradycardias & $\begin{array}{l}16.41 \\
( \pm 15.58)\end{array}$ & $\begin{array}{l}21.28( \pm \\
17.37)\end{array}$ & 0.39 & 8.26 & 0.15 \\
\hline Number of saturations & $\begin{array}{l}8.24 \\
( \pm 12.16)\end{array}$ & $\begin{array}{l}9.67( \pm \\
10.02)\end{array}$ & 0.71 & 2.31 & 0.53 \\
\hline $\begin{array}{l}\text { Weight difference between Day } 1 \text { and Day } \\
10(\mathrm{~g})\end{array}$ & $240( \pm 94)$ & $237( \pm 87)$ & 0.92 & -17.1 & 0.57 \\
\hline Discharge weight (g) & $2877( \pm 366)$ & $2821( \pm 474)$ & 0.70 & & \\
\hline Total hospital stay (days) & $\begin{array}{l}76.18 \\
( \pm 15.88)\end{array}$ & $\begin{array}{l}78.72 \\
( \pm 17.67)\end{array}$ & 0.66 & & \\
\hline
\end{tabular}

Data are presented as mean $( \pm S D)$.

Mean number of apneas, bradycardias and desaturations, per patient, during the 10 days of OFS : difference in weight between the first and tenth day of the OFS protocol; weight at discharge; total length of hospital stay in the experimental vs. the control group.

OFS: oro-facial stimulation; SD: Standard Deviation.

$p$ value of the difference between the groups with available data, $p<0.05$ : statistically significant difference.

a Value of $p$ in univariate analysis.

b Value of $\mathrm{p}$ in multivariate analysis including the parameters: Gestational Age, Birth Weight and total duration of ventilation.

ß: correlation coefficient of the multivariate analyses.

Table 4. Nutritional follow-up at 6 months, corrected age, and children's QUALIN quality of life score at 6 and 12 months of age, as assessed by parents (experimental vs. control group). 


\begin{tabular}{|llll|}
\hline Follow-up & $\begin{array}{l}\text { With OFS } \\
(\mathrm{n}=15)\end{array}$ & $\begin{array}{l}\text { Without OFS } \\
(\mathrm{n}=14)\end{array}$ & $\mathrm{p}^{\mathrm{a}}$ \\
\hline Number of breast feedings at 6 months (artificial/maternal) & $15(100) / 0$ & $14(100) / 0$ & $/$ \\
\hline Diversification at 6 months & $15(100)$ & $14(100)$ & $/$ \\
\hline Normal gag reflex at 6 months & $15(100)$ & $14(100)$ & $/$ \\
\hline Infant's quality of life as assessed by parents & & & \\
\hline 6 months QUALIN Score & $0.95 \pm 0.26$ & $0.90 \pm 0.18$ & 0.70 \\
\hline 12 months QUALIN Score & $1.06 \pm 0.24$ & $0.96 \pm 0.27$ & 0.36 \\
\hline
\end{tabular}

Data are presented as $n(\%)$ unless stated differently

OFS: oro-facial stimulation

QUALIN Questionnaire: quality of life score for infants and very young children, comprising 34 items. Twelve-month follow-up: experimental group $(n=13)$, control group $(n=14)$.

$\mathrm{p}$ : value of the difference between the groups with available data, obtained by $\chi 2$ test.

aValue of $p$ in univariate analysis.

$p<0.05$ : statistically significant difference.

\section{Figures}




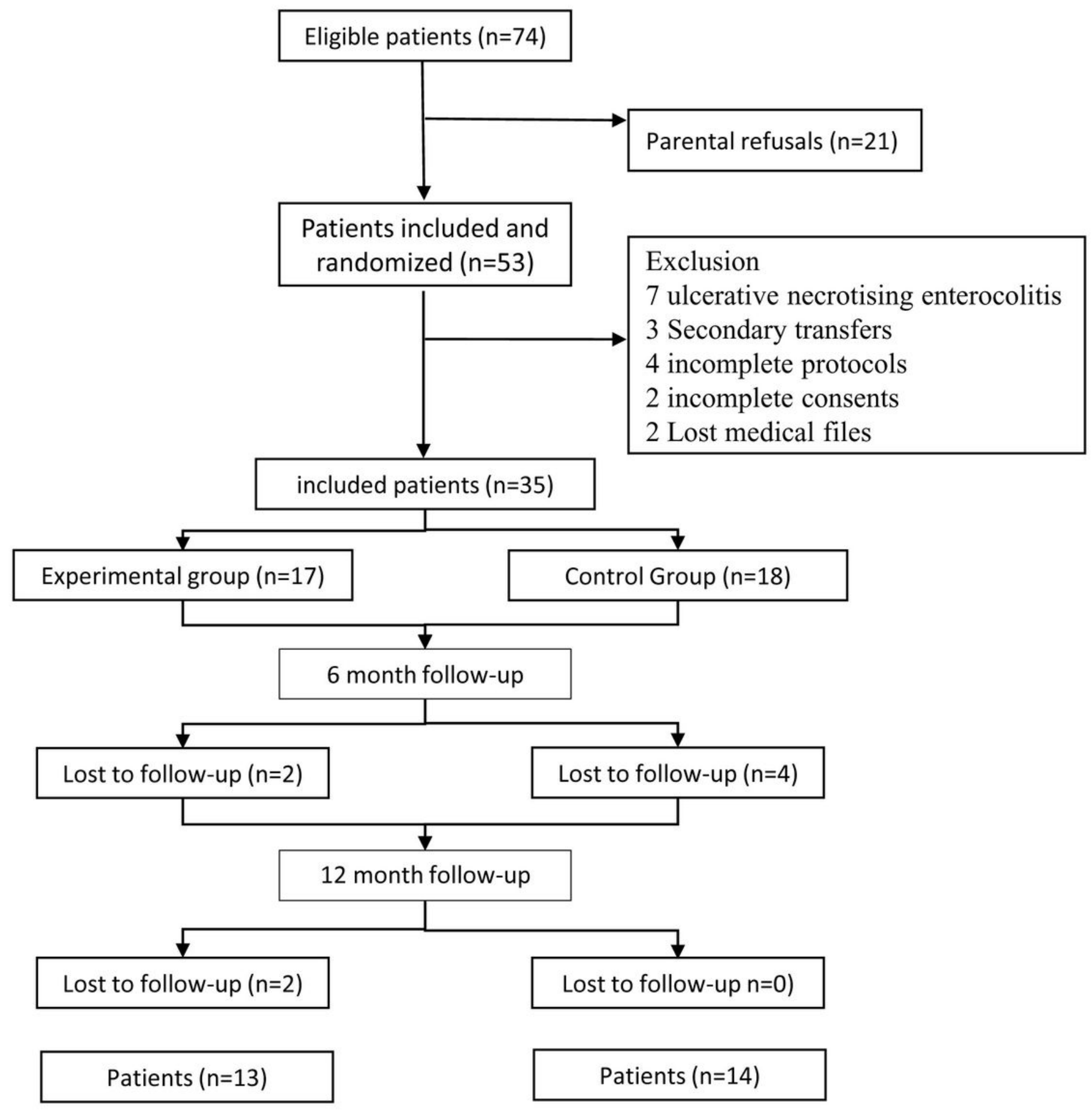

Figure 1

Flow chart 


\section{Quantity of milk ingested ( $\mathrm{ml}$ per day)}

800

700

600

500

400

300

200

100

0

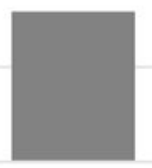

$$
\begin{gathered}
\text { First feeding } \\
\begin{array}{c}
{ }^{a} p=0,04^{*} \\
{ }^{b} p=0,07
\end{array}
\end{gathered}
$$

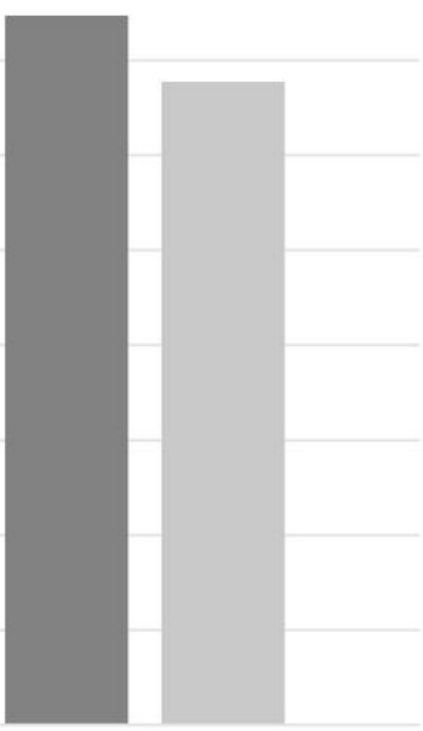

Four feeding

${ }^{a} \mathrm{p}=0,75$

Eight feeding

${ }^{a} p=0,34$

With OFS Without OFS

\section{Figure 2}

Amount of milk ingested during the 1st, 4th and 8th independent feeding $(\mathrm{ml})$, in the experimental (OFS $n=17$ ) vs. control group (without OFS, $n=18$ ). p: value of the difference between groups with available data a Value of $p$ in univariate analysis. $b$ Value of $p$ in multivariate analysis including Gestational Age, Birth Weight and total duration of ventilation. ${ }^{*} p<0.05$ : statistically significant difference. 


\section{FEEDING DATES (corrected age)}

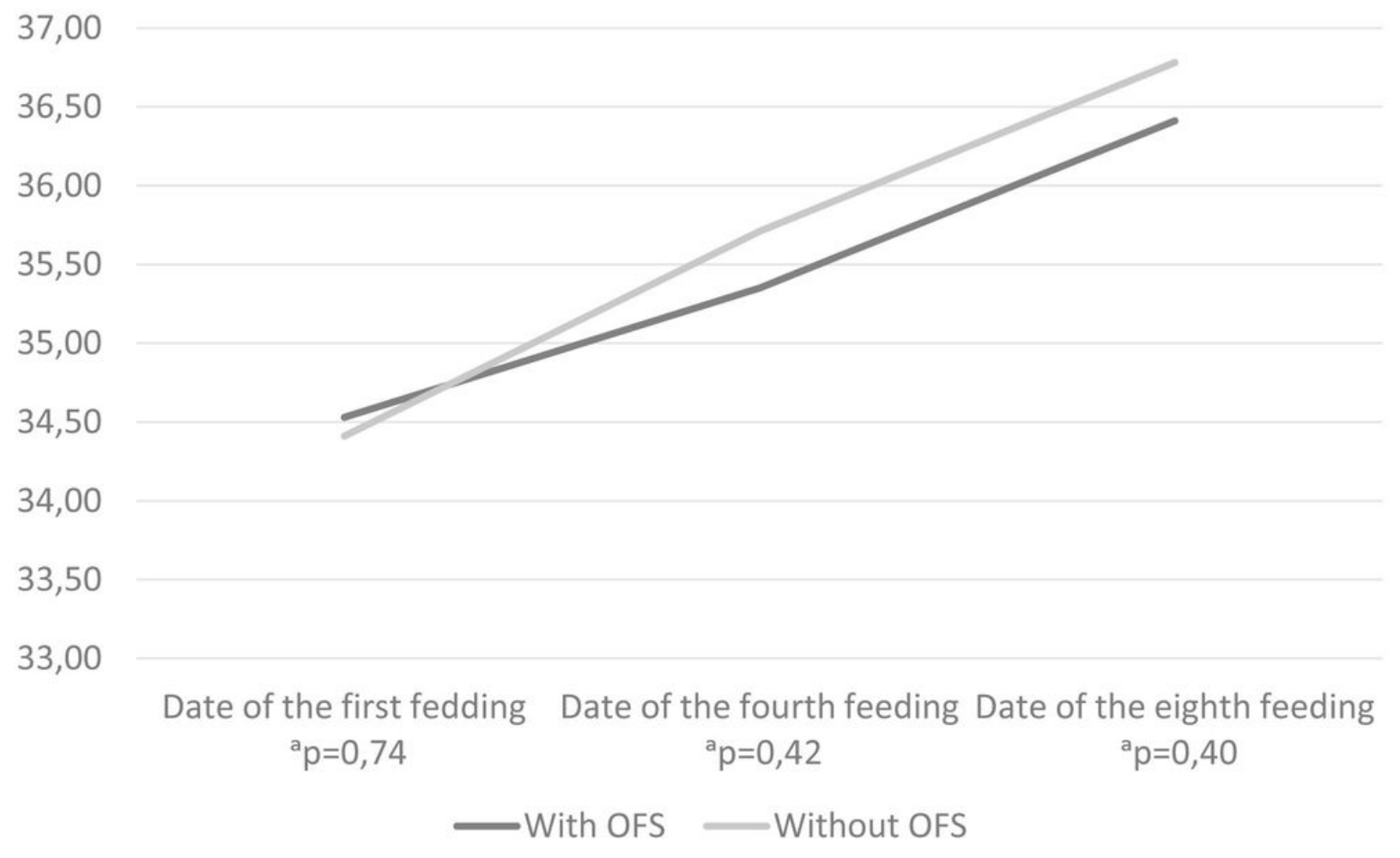

\section{Figure 3}

Experimental and control group dates of first, 4TH and 8TH independent feeds, Week GA-adjusted. Abbreviations: OFS: oro-facial stimulation. $p$ value of the difference between the groups with available data, $p<0.05$ : statistically significant difference. a Value of $p$ in univariate analysis. 